\title{
Read-out calibration of a SOI capacitive transducer using the pull-in voltage
}

\author{
L Mol $^{1}$, L A Rocha ${ }^{2}$, E Cretu ${ }^{3}$ and R F Wolffenbuttel ${ }^{1}$ \\ ${ }^{1}$ Delft University of Technology, Faculty of Electrical Engineering, EEMCS, Mekelweg 4, \\ 2828 CD Delft, The Netherlands \\ ${ }^{2}$ University of Minho, School of Engineering, Campus de Azurem, 4800-058 Guimaraes, Portugal \\ ${ }^{3}$ University of British Columbia, Department Electrical and Computer Engineering, 2332 Main Mall, \\ Vancouver, BC, V6T 1Z4, Canada \\ E-mail: 1.mol@tudelft.nl
}

Received 14 December 2007, in final form 6 March 2008

Published 13 May 2008

Online at stacks.iop.org/JMM/18/064009

\begin{abstract}
The pull-in voltage of a parallel plate electrostatic transducer is used to determine the amount of over-etching in fabricated devices. A detailed analysis of the capacitor behaviour over the full displacement range yields a model, which is used to describe the relation between over-etching and measured pull-in voltage. SEM photos confirm the over-etching measurement based on pull-in voltage. This information is used to linearize a charge amplifying read-out circuit with an intrinsic nonlinear transfer function. The remaining nonlinearity error of the read-out is less than $1 \%$.
\end{abstract}

(Some figures in this article are in colour only in the electronic version)

\section{Introduction}

Although micromachining enables the realization of very small features, the relative uncertainty in the dimensions is higher than in similar structures of more conventional macro-sized dimensions. Variations in dimensions of $10 \%$ are not uncommon [1,2]. This limits the usefulness of models based on mask dimensions, since actual dimensions will always deviate from mask dimensions, thereby causing changes in key parameters such as resonance frequency [2-4], sensitivity, displacement range and damping coefficient. A major cause for the uncertainty of the dimensions in many surface micromachining processes is over-etching $[5,6]$, which decreases beam widths, yet increases gap sizes.

One approach to circumvent the sensitivity to processinduced variations is to minimize the effect by design: the effect of dimensional uncertainty is modelled and relative device dimensions are chosen for minimized overall effect $[3,4]$. However, these methods are limited to certain classes of microstructures suitable for such modelling and do not guarantee complete immunity to process variations.

Another approach is to implement a technique for measuring process parameters after fabrication. Existing methods to determine fabricated device dimensions are often based on optical measurements, such as SEM imaging or laser interferometry [7-10]. These are costly and slow, hence not suited for use on a per device basis. Another possibility is to add extra microstructures specifically aimed at measuring process and device parameters [11]. This enlarges the chip area and adds to design complexity.

This paper presents an alternative to existing methods to estimate the amount of over-etching in 1-DOF electrostatic transducers based on the measurement of one single parameter, namely the well-defined pull-in voltage $[12,13]$. The amount of over-etching is consequently used to adjust the device model, which then accurately reflects the fabricated device properties. The adjusted device model then allows the linearization of a charge amplifying read-out circuit.

The ability to adjust the device modelling to account for process variation relies on the concurrent incorporation of an over-etch parameter in three sub parts of the model: the suspension spring, the capacitor gap and the electrostatic force due to an actuation voltage.

\subsection{The 1-DOF electrostatic transducer}

A 1-DOF accelerometer with separate drive and sense capacitors has previously been designed and used in several 


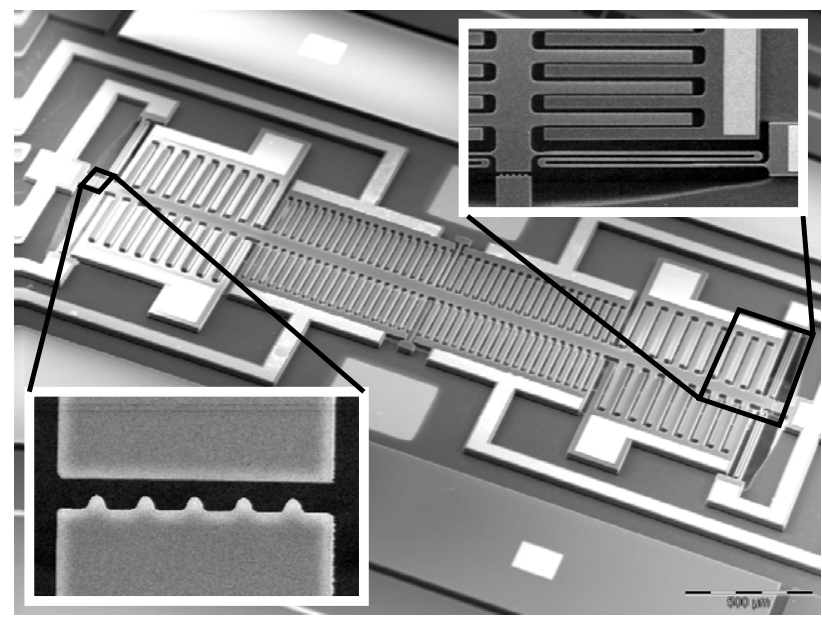

Figure 1. SEM image of the fabricated device. The narrow electrodes in the centre form the sensing capacitors, the wider ones on the outside are for electrostatic actuation. Insets show details of a spring, a stopper and actuation electrodes.

experimental projects related to inertial MEMS (the pull-in instability [12, 13], squeezed-film damping measurements [14] and nonlinear control techniques [15]). To obtain more precise and extensive measurement data, a new device was designed and fabricated (figure 1) to overcome some of the limitations associated with the first generation devices. For this design a new fabrication process is used, to enable the realization of thicker $(25 \mu \mathrm{m}$ instead of $10 \mu \mathrm{m})$ and higher aspect-ratio devices. The main benefit of the increased thickness is the larger proof mass at given lateral dimensions and, therefore, the increased sensitivity. In addition, a higher aspect ratio increases the feasible capacity per unit chip area.

\section{Fabrication process}

The transducer is fabricated in a multi-user silicon-on-insulator (SOI) micromachining process offered by MEMSCAP, which is a four-mask-level patterning and etching process [16]. The starting wafer is a stack of a $400 \mu \mathrm{m}$ substrate layer, a $1 \mu \mathrm{m}$ oxide layer and a $25 \mu \mathrm{m}$ silicon layer. The silicon layer is patterned and subsequently reactive ion etched (DRIE) using inductively coupled plasma. After application of a front side protection layer the wafer is reversed and the substrate layer is patterned. A DRIE etch step etches up to the oxide layer, after which the front side protection layer is stripped again. Then all exposed oxide is removed.

Two metal mask steps allow for fine and coarse metal features; however, no means of crossing interconnect or making vias is available. This limits structures to purely planar topologies.

Figure 2 illustrates the process showing a cross section of the device at one of the springs and the first two of the set of actuation electrodes.

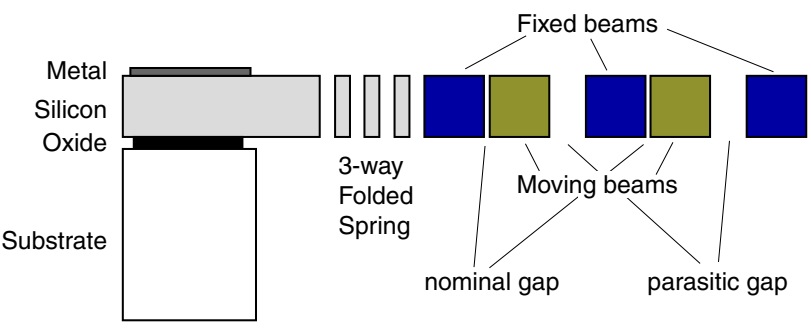

Figure 2. Cross sectional view of part of the fabricated device (according to inset in figure 1) showing the layers of the SOI mumps process, one of the four folded springs and the electromechanical configuration of the actuation beams.

Table 1. Overview of designed and actual device parameters.

\begin{tabular}{lcl}
\hline & Value by design & Actual value \\
\hline Mass & $42 \mu \mathrm{g}$ & \\
Small gap size $d_{n}$ & $2 \mu \mathrm{m}$ & $2.92 \mu \mathrm{m}$ \\
Spring constant $k$ & $14.8 \mathrm{~N} \mathrm{~m}^{-1}$ & $8.85 \mathrm{~N} \mathrm{~m}^{-1}$ \\
Vpull-in & $5.42 \mathrm{~V}$ & $7.45 \mathrm{~V}$ \\
\hline
\end{tabular}

\section{Device design and operational parameters}

A 1-DOF accelerometer with separate drive and sense capacitors was designed and fabricated (figure 1). The device consists of a laterally movable mass of $42 \mu \mathrm{g}$ that is suspended on springs from the substrate. Several electrodes on both sides of the suspended beam structure are interleaved with electrodes that are mechanically anchored to the substrate. Since the fabrication process does not provide a means for crossing interconnect metal only planar topologies are supported. This complicates the realization of differential capacitive structures. The problem is circumvented by placing the movable electrodes asymmetrically within the gaps formed by the fixed electrodes. Figure 3 shows the device conceptually as well as its equivalent electrical representation.

\subsection{Actuation and sense electrodes}

A total of 18 wide electrode sets combined form a driving capacitor for electrostatic actuation of the device to one side. Each individual beam is $300 \mu \mathrm{m}$ long and $25 \mu \mathrm{m}$ wide. Another set serves the same purpose for the other direction. Forty narrow beams per side form the sense capacitors. Each beam is $230 \mu \mathrm{m}$ long and $10 \mu \mathrm{m}$ wide. The smallest gap size for a device in the initial position is $2 \mu \mathrm{m}$ for both the sense and actuation capacitors.

\subsection{Stoppers}

Stoppers have been designed at the fixed landing pad of the movable beam. These prevent complete and irreversible stiction of the device in the event of pull-in (snap down) due to electrostatic force or excessive inertial force. The individual actuation beams must be rigid enough to prevent contact due to excessive bending in case of a pull-in event with the gap size decreased to $0.25 \mu \mathrm{m}$ (defined by the stopper dimensions). These are, therefore, wider than the beams of the sense electrode array. 

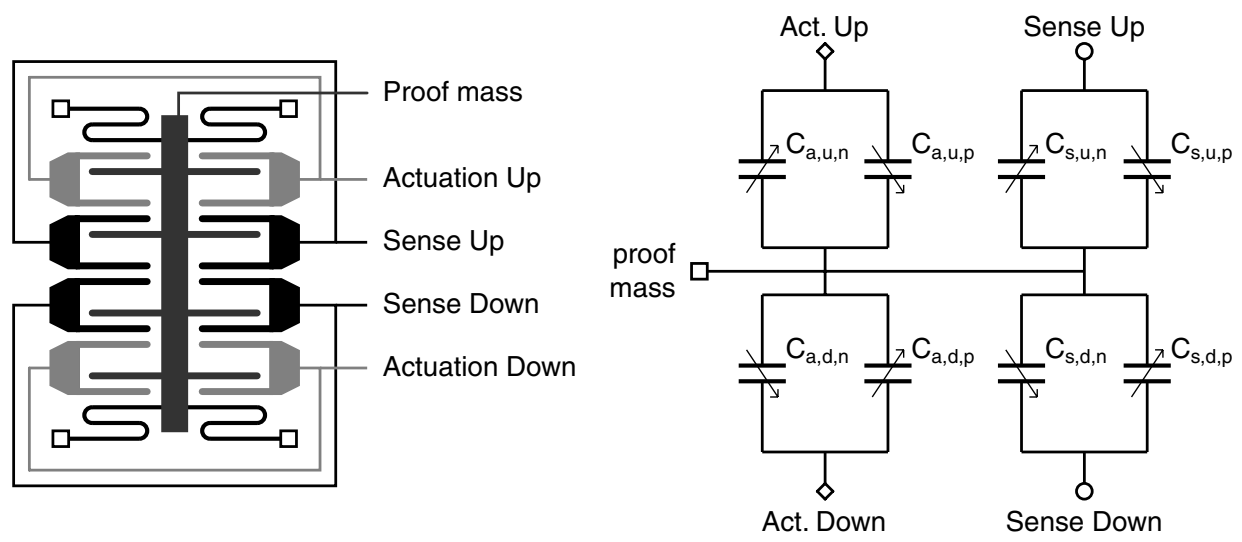

Figure 3. Schematic layout of the device and its electrical equivalent.

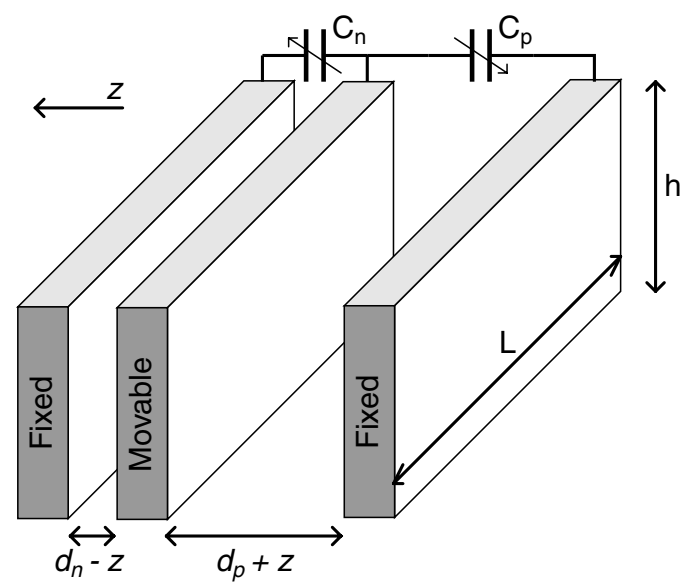

Figure 4. The generic asymetric capacitive finger configuration.

\section{Modelling}

In order to predict the static and dynamic behaviour of the transducer a model is needed that includes its mass, sense and actuation capacities, spring forces as well as gas damping forces and electrostatic forces, all as a function of displacement. To use the model for an actually fabricated device it must also cope with process variation induced changes in dimension.

\subsection{Capacity and electrostatic force}

Consider the electrode layout as presented in figure 4, where the fixed electrodes are at the same potential. For $L \gg h$ only the limited aspect ratio of $h / d$ contributes to the effect of fringe fields.

The fringe fields can be accounted for using a correction factor $G_{\mathrm{ff}}$ based on the ratio $h / d[17,18]$ :

$$
\begin{aligned}
& G_{\mathrm{ff}}=\frac{\left(1+\ln \left(\frac{2 \pi h}{\mathrm{~d} z}\right)\right) \mathrm{d}(z)}{\pi h} \\
& d(z)=\left\{\begin{array}{ll}
d_{n}-z \\
d_{p}+z
\end{array} \quad \text { for } \quad \begin{array}{l}
\text { Nominal } C_{n} \\
\text { Parasitic } C_{p} .
\end{array}\right.
\end{aligned}
$$

Additionally for practical ratios $10>d_{p} / d_{n}>1$, the contribution of the parastic $C_{p}$ to the total capacity $C_{t}=$

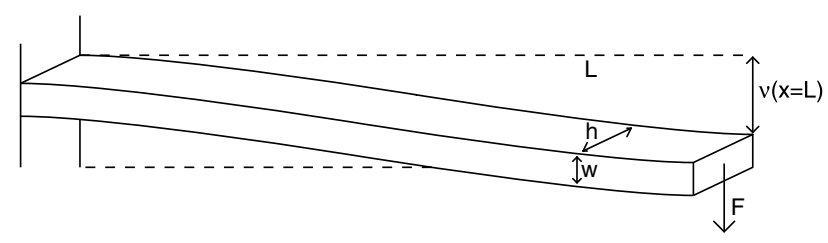

Figure 5. A beam clamped at one side and free to translate on the other side when subjected to a loading force.

$C_{n}+C_{p}$ is significant and cannot be neglected, especially for displacements $z<0$ where the nominal gap is widening and the parasitic gap becomes smaller.

The electrostatic force is a function of the derivative of capacity to displacement $z$

$$
\begin{aligned}
F_{\mathrm{es}}=\frac{1}{2} \frac{\partial C(z)}{\partial z} V^{2} & =\frac{1}{2}\left(\frac{\partial C_{n}(z)}{\partial z}+\frac{\partial C_{p}(z)}{\partial z}\right) V^{2} \\
C_{n}(z) & =G_{\mathrm{ff}}(z) \varepsilon_{0} \frac{h L}{d_{n}-z} \\
C_{p}(z) & =G_{\mathrm{ff}}(z) \varepsilon_{0} \frac{h L}{d_{p}+z}
\end{aligned}
$$

and is used further on to calculate the pull-in voltage.

\subsection{Spring force}

Consider a prismatic beam of with $w$ and height $h$, with flexural rigidity $E I_{x}$ and length $L$. One end is fixed (no rotation, no translation), whilst the free tip is loaded with force $F$ and constrained to translation only (figure 5). The bending moment equation for such a beam is

$$
E I_{x}\left(\frac{\mathrm{d}^{2}}{\mathrm{~d} x^{2}} v(x)\right)=\frac{F(L-2 x)}{2} .
$$

Integrating twice and solving the integration constants using boundary conditions yields an expression for the deflection curve $v(x)$ with $x$ being the position along the beam:

$$
v(x, F)=-\frac{F x^{2}(-3 L+2 x)}{12 E I_{x}} .
$$




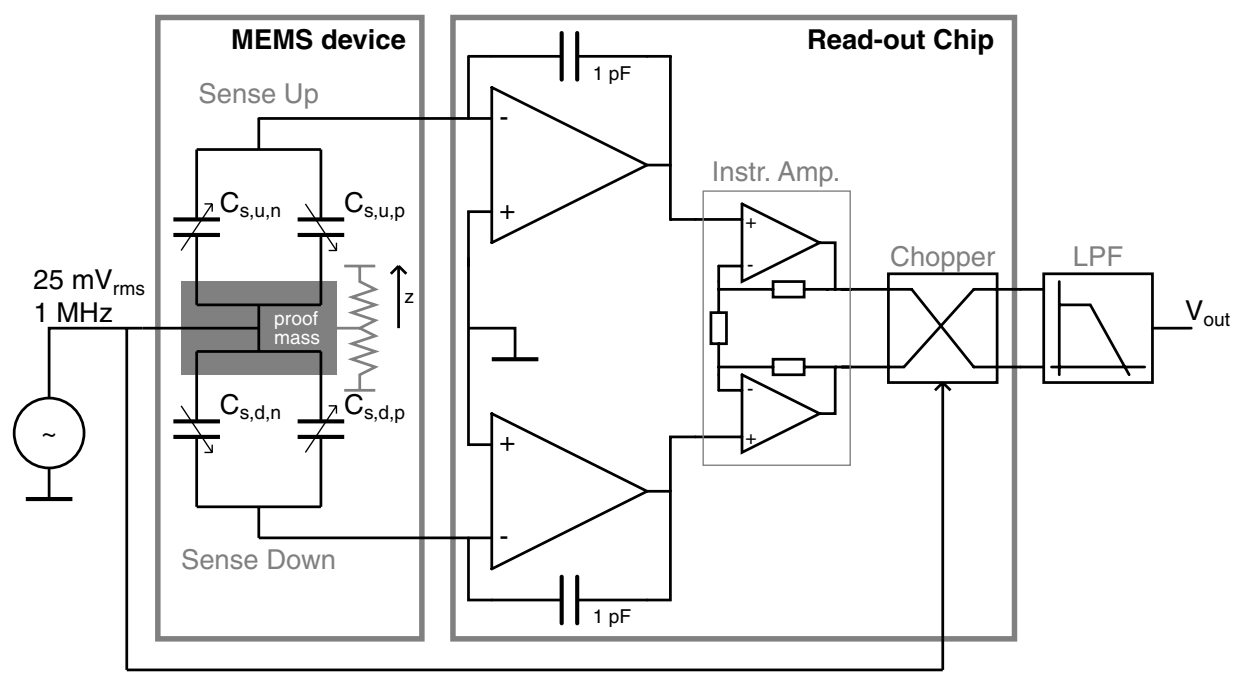

Figure 6. Simplified schematic of the total readout circuit. The displacement readout is implemented with a two-channel differential charge amplifier circuit and a coherent detection mixer realized in a single custom $0.35 \mu \mathrm{m}$ CMOS chip. The actuation capacitors of the MEMS device are not shown.

From the maximum deflection at the tip $(x=L)$ the equivalent spring constant is then found as

$$
k_{\text {beam }}=\frac{12 E I_{x}}{L^{3}}
$$

with $I_{x}$ being the moment of inertia dependent on the cross section dimensions $h$ and $w$ :

$$
I_{x}=\frac{h w^{3}}{12} .
$$

In this particular device the suspension of the movable mass consists of four identical springs. Each spring is a three-way folded beam. For each beam section a spring constant $k$ can be calculated according to (5). The total equivalent spring constant for the proof mass suspension is therefore

$$
k_{\mathrm{total}}=\frac{4 k_{\mathrm{beam}}}{3} .
$$

\subsection{Pull-in voltage}

The electrostatic force resulting from an applied voltage is counteracted by the spring force $\mathrm{z} \cdot k_{\text {total }}$ and must be equal for static equilibrium (with $z$ the electrode displacement). Additionally at the critical pull-in point the derivative of the electrostatic force to displacement $z$ is equal to the spring constant $k_{\text {total }}$.

$$
\begin{array}{r}
F_{\text {total }}=F_{\text {es }}+k_{\text {total }} z=0 \\
\\
\frac{\partial F_{\text {es }}(z)}{\partial z}=k_{\text {total }} .
\end{array}
$$

By solving these equations the pull-in voltage for given device dimensions can be found [13].

\subsection{The read-out circuit}

For position read-out a custom $0.35 \mu \mathrm{m}$ CMOS circuit is designed and implemented. The microstructure and the readout circuit are wire bonded to a printed circuit board. The microstructure is used as a differential sense capacitor in a two-channel differential charge amplifier. A high-frequency carrier signal is connected to the proof mass and is amplitude modulated by displacement of the movable electrode (i.e. the proof mass). The difference signal of the two charge amplifiers is amplified in an instrumentation amplifier and fed to a chopper that coherently demodulates the signal to a dc level using the carrier signal. The differential current output is converted to a voltage output and an instrumentation amplifier shifts the signal level to quasi dc around $0 \mathrm{~V}$. The topology of the readout circuit is schematically shown in figure 6 .

The advantage of the symmetric current mode topology compared to voltage readout is reduced cross sensitivity to unwanted stray capacitance. However, the output voltage of the used readout circuit is not a linear function of displacement, due to this input configuration. The problem of the nonlinear read-out is overcome by using the previously derived model for the capacity as a function of displacement. An analytic expression for the correcting scaling factor can be derived by

$C_{\text {diff }}(z)=C_{s, u, n}(z)+C_{s, u, p}(z)-C_{s, d, n}(z)-C_{s, d, p}(z)$

$A_{c f}(z)=\frac{C_{\mathrm{diff}}(z)}{z\left(\left.\frac{\partial C_{\mathrm{diff}}(z)}{\partial z}\right|_{z=0}\right)}$.

Using this correction factor, the output voltage of the readout circuit is now a linear function of the displacement of the movable electrode.

\section{Measuring over-etch through pull-in voltage}

Due to technological imperfections and process variations the designed device dimensions will never be exactly fabricated. A 


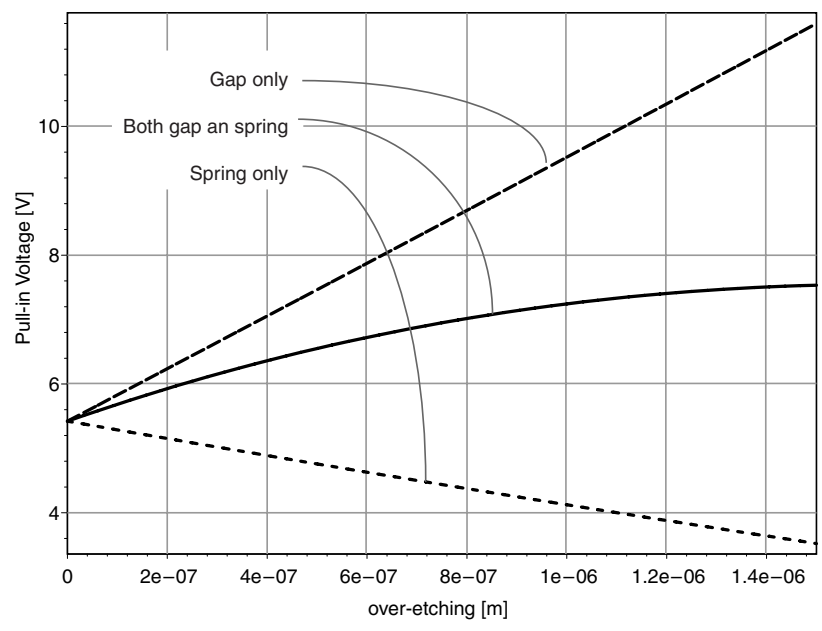

Figure 7. Typical influence of the amount of over-etching on the pull-in voltage due to weaker spring, smaller capacity and both effects combined.

common effect in DRIE process steps is over-etching. This has a strong effect on two device parameters: (a) spring constant and (b) sense and actuation capacities. For a given amount of over-etch (which we consider uniform), the spring becomes less stiff which will lower the pull-in voltage, whereas the capacity gets smaller and has an opposing (i.e. increasing) effect on the pull-in voltage. This means the influence of overetching is not decoupled. Figure 7, shows both effects and the combined effect and from this figure it becomes clear that the pull-in voltage can effectively be used to determine the amount of over-etching.

Given a measured pull-in voltage for a fabricated device the amount of over-etch can be found for which measured device parameters correspond to modelled device parameters. A Maple worksheet is used to solve a set of equations that integrally model the concurrent effect of over-etching on the spring stiffness and the capacitor values (i.e. equations (1), (2), (7) and (8) with added over-etching variable). In doing so, the maple worksheet finds the amount of over-etching that corresponds to the measured pull-in voltage. From the pull-in voltages the accurate estimates of the actual fabricated dimension are now known and used accordingly in the device model.

\section{Experimental results}

Figure 8 shows the measured readout voltage as a function of actuation voltage for both displacements 'up' and 'down'. Note that only the pull-in voltage can be determined, but no quantative data on the displacement is available from this measurement. Using the Maple worksheet to solve the set of equations the amount of over-etch is determined to be $0.92 \mu \mathrm{m}$. SEM images of the microstructures confirm this to be a typical value for the device.

The actual initial gap size and spring constant are now known. Using these actual device dimensions the correction factor needed to linearize the read-out circuit is now found. Thus the displacement can be calculated for

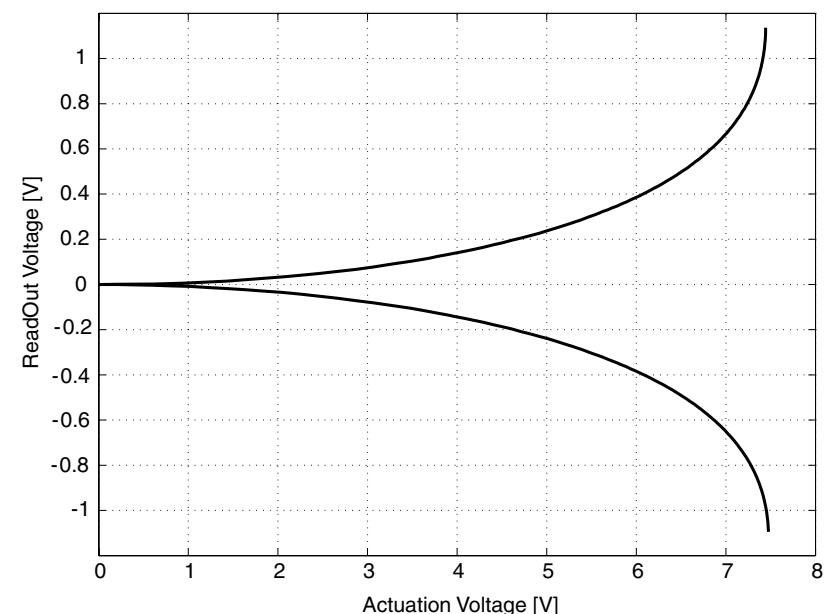

Figure 8. Measured output voltage as a function of actuation voltage for two directions of actuation.

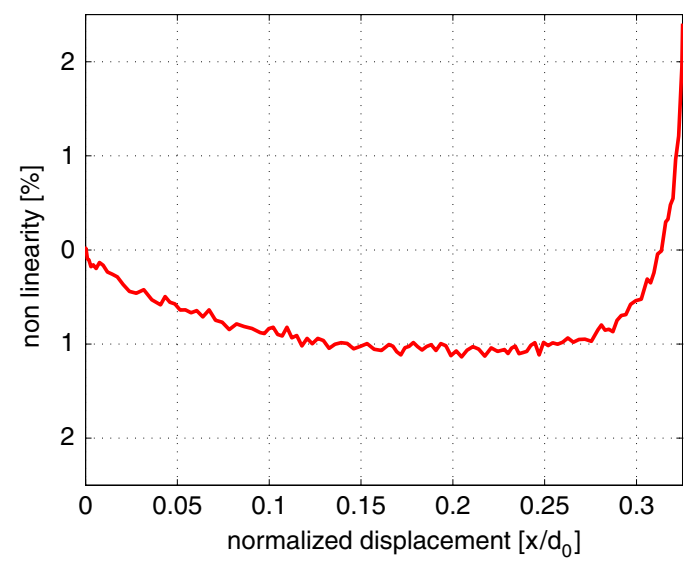

Figure 9. Residual nonlinearity error of the displacement to read-out voltage relation.

a given applied voltage, which in turn serves as an input to calculate the correction factor for the readout voltage. Using the nonlinearity correction factor and the model based on the estimated fabricated device dimensions yields a nonambiguous relation between actuation voltage, displacement and read-out circuit voltage.

The residual nonlinearity error in the relation between displacement due to electrostatic actuation and read-out voltage is shown in figure 9.

The largest residual error occurs at one-third of the initial gap (the static pull-in point). At that point the model is very sensitive to small changes in the pull-in voltage of a device.

\section{Conclusions and future work}

Neither the influence of over-etching on capacity nor on spring stiffness can be neglected in accurate modelling of an electrostatic transducer. By extracting information on the amount of over-etching from the measured pull-in voltage of an electrostatic transducer a compact simulation model conforming to fabricated dimensions can be obtained. This 
model enables the linearization of the readout circuit with a residual error less than $1 \%$ almost up to the static pull-in point.

Using a closed loop control to extend the travel range of the movable mass beyond the static pull-in point is expected to yield even lower residual nonlinearity errors over the full displacement range.

\section{Acknowledgments}

Parts of this work would not have been possible without the assistance of DIMES (the Delft Institute of Microsystems and Nanoelectronics).

\section{References}

[1] Dewey A, Ren H and Zhang T H 2000 Behavioral modeling of microelectromechanical systems (MEMS) with statistical performance-variability reduction and sensitivity analysis IEEE Trans. Circuits Syst. 47 105-13

[2] Hong Y S, Lee J H and Kim S H 2000 A laterally driven symmetric micro-resonator for gyroscopic applications J. Micromech. Microeng. $10452-8$

[3] Liu R, Paden B and Turner K 2002 Mems resonators that are robust to process-induced feature width variations J. Microelectromech. Syst. 11 505-11

[4] Mawardi A and Pitchumani R 2005 Design of microresonators under uncertainty J. Microelectromech. Syst. 14 63-9

[5] Chen B T and Miao J M 2007 Influence of deep rie tolerances on comb-drive actuator performance J. Phys. D: Appl. Phys. 40 970-6

[6] Li J, Liu A Q and Zhang Q X 2006 Tolerance analysis for comb-drive actuator using drie fabrication Sensors Actuators A 125 494-503

[7] Burdess J S, Harris B J, Wood D, Pitcher R J and Glennie D 1997 A system for the dynamic characterization of microstructures J. Microelectromech. Syst. 6 322-8
[8] Burns D J and Helbig H F 1999 A system for automatic electrical and optical characterization of microelectromechanical devices J. Microelectromech. Syst. 8 473-82

[9] Brown G C and Pryputniewicz R J 2000 New test methodology for static and dynamic shape measurements of microelectromechanical systems Opt. Eng. 39 127-36

[10] Hart M R, Conant R A, Lau K Y and Muller R S 2000 Stroboscopic interferometer system for dynamic mems characterization J. Microelectromech. Syst. 9 409-18

[11] Osterberg P M and Senturia S D 1997 M-test: a test chip for mems material property measurement using electrostatically actuated test structures J. Microelectromech. Syst. $6107-18$

[12] Tilmans H A C and Legtenberg R 1994 Electrostatically driven vacuum-encapsulated polysilicon resonators: 2 . Theory and performance Sensors Actuators A 45 67-84

[13] Rocha L A, Cretu E and Wolffenbuttel R F 2004 Analysis and analytical modeling of static pull-in with application to mems-based voltage reference and process monitoring J. Microelectromech. Syst. 13 342-54

[14] Mol L, Rocha L A, Cretu E and Wolffenbuttel R F 2006 Experimental verification of rarefied gas squeezed-film damping models used in mems ASME Intern. Mech. Eng. Conf. and Exh. (Chicago)

[15] Rocha L A, Cretu E and Wolffenbuttel R F 2006 Using dynamic voltage drive in a parallel-plate electrostatic actuator for full-gap travel range and positioning J. Microelectromech. Syst. 15 69-83

[16] Miller K, Cowen A, Hames G and Hardy B 2004 Soimumps Design Handbook http://www.memsrus.com/ documents/SOIMUMPs.dr.v4.pdf

[17] Palmer H B 1937 Capacitance of a parallel-plate capacitor by the Schwartz-Christoffel transformation Trans. AIEE 56 363-6

[18] Leus V and Elata D 2004 Fringing field effect in electrostatic actuators TECHNION ETR-2004-2 Israel Institute of Technology 